\title{
The core fundamental plane of B2 radio galaxies
}

\author{
D. Bettoni ${ }^{1}$, R. Falomo ${ }^{1}$, P. Parma ${ }^{2}$, H. de Ruiter ${ }^{3,2}$, and R. Fanti ${ }^{2}$ \\ 1 INAF - Osservatorio Astronomico di Padova, Vicolo dell'Osservatorio, 5, 35122 Padova, Italy \\ e-mail: [daniela.bettoni; renato.falomo]@oapd.inaf.it \\ 2 INAF - IRA Bologna, Italy \\ e-mail: [p.parma;r.fanti]@ira.inaf.it \\ 3 INAF - Osservatorio Astronomico di Bologna, via Ranzani, 1, 40127 Bologna, Italy \\ e-mail: h.deruiter@ira.inaf.it
}

Received 3 August 2009 / Accepted 3 October 2009

\begin{abstract}
Context. The photometric, structural and kinematical properties of the centers of elliptical galaxies harbour important information on the formation history of galaxies. In the case of non-active elliptical galaxies, these properties are linked in a way that surface brightness, break radius and velocity dispersion of the core lie on a fundamental plane similar to that found for their global properties. Aims. We construct the core fundamental plane (CFP) for a sizeable sample of low redshift radio galaxies and compare it with that of non-radio ellipticals.

Methods. We combine data obtained from high resolution HST images with medium resolution optical spectroscopy to derive the photometric and kinematic properties of $\sim 40$ low redshift radio galaxies.

Results. We found that the CFP of radio galaxies is indistinguishable from that defined by non-radio elliptical galaxies of similar luminosity. The characteristics of the CFP of radio galaxies are also consistent (same slope) with those of the fundamental plane (FP) derived from the global properties of radio (and non-radio) elliptical galaxies. The similarity of CFP and FP for radio and non-radio ellipticals suggests that the active phase of these galaxies has minimal effects on the structure of the galaxies.
\end{abstract}

Key words. galaxies: active - galaxies: kinematics and dynamics

\section{Introduction}

The properties of the centers of massive elliptical galaxies are of strategic importance for the understanding of the complex processes of galaxy formation. The centers represent the bottom of the potential well of the galaxy, host massive black holes, and provide a record of the past history of the galaxies. Until a decade ago, the properties of the center of galaxies were very little studied because of the insufficient spatial resolution of the available instrumentation. Only with the use of the Hubble Space Telescope, and using future large ground based telescope, can such a study become feasible.

In the pioneering study on the nuclear properties of nearby early type galaxies (carried out with HST images), Faber et al. (1997) were able to probe the inner regions of a number of nearby ellipticals. They point out that the inner luminosity profiles can be parameterized by a core (or break) radius $R_{\mathrm{b}}$ and a characteristic surface brightness within the break radius $\left\langle\mu_{\mathrm{b}}\right\rangle$. They also showed that these parameters $\left(R_{\mathrm{b}},\left\langle\mu_{\mathrm{b}}\right\rangle\right)$ can be combined to form a core fundamental plane (CFP) which is analogous to the one found for the global properties of early type galaxies (Faber et al. 1997). Assuming that the cores are in dynamical equilibrium and supported by random motion, and that the velocity anisotropy does not vary too much from galaxy to galaxy, and if the core $M / L$ is a well-behaved function of any two variables $\left\langle\mu_{\mathrm{b}}\right\rangle, R_{\mathrm{b}}$, or $\sigma_{0}$, then one expects that the cores of galaxies follow a law similar to that of the fundamental plane (FP).

The main reason to study global and core properties is that it allows one to probe the mechanism of galaxy formation. In the last decade a new tool has become available: the discovery of the presence of a massive black bole (BH) in the centers of virtually all galaxies (e.g. Ferrarese \& Merritt 2000; Lauer et al. 2007). However, only a small fraction of these BHs exhibit associated nuclear activity (non-thermal nuclear emission, $\mathrm{X}$-ray and radio emission). The BHs may play an important role in the formation and evolution of massive galaxies and are also a key component for the development of the nuclear activity. Therefore the comparison of the properties of active and inactive galaxies through the FP becomes a tool to investigate the interplay between galaxy formation and nuclear activity.

In a previous work, using photometrical and dynamical data for 73 low red-shift $(z<0.2)$ radio galaxies $(\mathrm{RG})$, we (Bettoni et al. 2001) were able to compare the FP of RG with the one defined by inactive ellipticals (Jørgensen et al. 1996, JFK96). We showed that the same FP holds for both radio and non-radio ellipticals with radio galaxies occupying the region of the most luminous and large galaxies.

Until very recently little data have been available on the optical nuclear properties of radio galaxies. One of the best optical sets of data is the study of the B2 sample of low luminosity radio galaxies (Fanti et al. 1987; Capetti et al. 2000). For $\sim 60$ radio galaxies WFPC2 HST imaging is available in the $F 555 W$ (approximately $V$ ) and $F 814 W$ (approximately I) filters. A similar set of data is also available for a sample of powerful 3C radio sources. These studies revealed the presence of new and interesting features, some of them almost exclusively associated with low luminosity FR I radio galaxies. In particular, the HST observations have shown the presence of dust in a large fraction of weak (FR I) 
radio galaxies which takes the form of extended nuclear disks (Jaffe et al. 1993; De Koff et al. 1996; De Juan et al. 1996; Verdoes Kleijn et al. 1999; de Ruiter et al. 2002). Such structures have been naturally identified with the reservoir of material which will ultimately accrete into the central black hole. The symmetry axis of the nuclear disk may be a useful indicator of the rotation axis of the central black hole (see e.g. Capetti \& Celotti 1999), although the precise relationship between these two axes remains uncertain.

In this paper we investigate the CFP for a sample of low redshift radio galaxies and to compare it with that for radio quiet galaxies. The plan of the paper is as follows. In Sect. 2 we discuss our observations and data reduction methods. In Sect. 3 we present the CFP for our sample of RG. The implications of those observations are then discussed in Sect. 4. Throughout this paper we use the Concordance Cosmological Model, with $H_{0}=70 \mathrm{~km} \mathrm{~s}^{-1} \mathrm{Mpc}^{-1}$, and $\Omega_{\Lambda}=0.70$.

\section{The sample of radio galaxies}

One of the most complete and well studied samples of nearby radio galaxies in the northern hemisphere is the B2 sample (Fanti et al. 1987). This sample consists of $\sim 100$ early-type galaxies and was extensively studied at radio wavelengths, especially since the 1980s (see Fanti et al. 1987; Parma et al. 1987; de Ruiter et al. 1990; Morganti et al. 1997). The sample is complete down to $0.25 \mathrm{Jy}$ at $408 \mathrm{MHz}$ and down to (roughly) $m_{v}=16.5$ and should be essentially unbiased for orientation. The objects span the radio power range between $10^{23}$ and $10^{26} \mathrm{~W} \mathrm{~Hz}^{-1}$ at $1.4 \mathrm{GHz}$ with a pronounced peak around $10^{25} \mathrm{~W} \mathrm{~Hz}^{-1}$. Therefore they give an excellent representation of the radio source types encountered below and around the break of the radio luminosity function.

We considered here the sub sample of the B2 sample $(\sim 100$ objects $)$ of 57 low redshift $(z<0.20)$ radio galaxies, for which photometric and structural data of the core are available from HST observations in the $F 814 W$ band (de Ruiter et al. 2002; Capetti et al. 2000). As described in Capetti et al. 2000 there is no bias in the selection of the 57 objects observed with HST (they were chosen randomly as far as their radio and optical properties are concerned). In comparing various parameters of this observed sub-sample with those of the sources that were not observed by HST they found that no significant differences emerged. The HST observations are complete at the level of $\sim 57 \%$ and therefore constitute an unbiased sub sample.

\section{Summary of previous results on the core properties of radio galaxies}

In de Ruiter et al. (2002) the dust properties of radio cores were analysed. About half of the sources have significant amounts of dust in the nuclear region, mostly in the form of disks or lanes, and if radio jets are present they tend to be perpendicular to the dusty disk or lane, at least in the low power sources $\left(P<10^{24} \mathrm{~W} \mathrm{~Hz}^{-1}\right.$ at $\left.1.4 \mathrm{GHz}\right)$. There is also a (broad) correlation between total dust mass of the disks and the radio power.

Based on high resolution HST images it was found that elliptical galaxies come in two flavours, one with "core" radial brightness profiles and one with power law profiles (see e.g. Faber et al. 1997). This distinction is seen also for other properties: rotation, isophote shape and presence of X-ray emission (see for a recent discussion Kormendy et al. 2009).
This dichotomy extends also to radio properties. Radial brightness profiles of sources with a moderate content of nuclear dust (de Ruiter et al. 2005) are of core type. This has been confirmed by Balmaverde \& Capetti (2006) \& Capetti \& Balmaverde (2006). Observations suggest that there is a class of early-type galaxies that will never harbour a classical radio source of the Fanaroff-Riley type I or II. These galaxies have steep power law inner profiles, with a slope (inner Nuker law) $\gamma>0.3$. On the other hand galaxies with a core profile $(\gamma<$ 0.3 ) may or may not possess a nuclear radio source. This difference seems genuine and not induced by selection effects. The absolute magnitudes of core and power law galaxies overlap and the power law galaxies are never associated with classical radio sources. This result is consistent with the dichotomy described above and suggests that AGN activity in core galaxies can be very pronounced and that the dichotomy is the result of strong or weak AGN feedback respectively (Kormendy et al. 2009).

\section{Stellar velocity dispersion}

To derive the CFP we need, together with the photometric data, the central velocity dispersion of the galaxy. For 11 galaxies in the sample, we found measurements of the stellar velocity dispersion in the literature, using the Lyon-Meudon Extragalactic Database (LEDA Paturel et al. 1997). These measurements of $\sigma$ were corrected to a circular aperture with a metric diameter of $1.19 h^{-1} \mathrm{kpc}$, equivalent to $3.4^{\prime \prime}$ at the distance of the Coma cluster. To derive $\sigma_{0}$ for the remaining sources, we carried out spectroscopic observations. We were able to observe 27 out of the remaining 46 galaxies. One object $(0648+27)$ was not included in the final sample to derive the CFP, as explained in Appendix A1. For this reason the final sample considered in this study is composed of 37 objects.

\subsection{Spectroscopic observations}

Optical spectroscopy in the range $\lambda \lambda=3700-4700 \AA$ and 4600-6500 $\AA$ was obtained in service mode in two observing runs in 2005 and 2006 with the Telescopio Nazionale Galileo (TNG). We used the spectrograph DOLORES equipped with a Loral CCD with $2048 \times 2048$ pixels of $15 \mu$. In 2005 we used the HRV Grism \#6 with a slit of 1.0 arcsec, this yields a velocity dispersion resolution $\Delta \sigma=75 \mathrm{~km} \mathrm{~s}^{-1}$. In 2006 we used the grism VHR-V with a slit of 1.0 arcsec, this yields a $\Delta \sigma=85 \mathrm{~km} \mathrm{~s}^{-1}$. The plate scale across the dispersion is $0.275 \mathrm{arcsec} / \mathrm{pix}$. In addition to the radio galaxy spectra, we secured spectra of bright stars of spectral type from G8III to K1III with low rotational velocity, $\left(V \times\right.$ seni $\left.<17 \mathrm{~km} \mathrm{~s}^{-1}\right)$. These spectra were used as templates of zero velocity dispersion. During the second observing run an early- type galaxy (NGC 3377), as standard for the measurement of $\sigma$, was also observed. The slit was oriented along the apparent major axis for all the galaxies in our sample except in the cases of galaxies with multiple nuclei, where the slit was aligned along the two nuclei. All the spectra were bias and flatfield corrected, trimmed and wavelength calibrated using standard procedures available in the IRAF package. The accuracy of the latter procedure was checked with measurements of the night sky $\lambda_{0}=5577.32 \AA$ emission line. The systemic velocity, corrected to the Sun, and the velocity dispersion $\sigma$ were determined using the Fourier Quotient method (Sargent et al. 1977; Bertola et al. 1994). The Fourier Quotient method was applied, using all the template stars, to all spectra to obtain the radial velocity and the velocity dispersion. The rms of the determinations 
Table 1. The sample of low redshift B2 radio galaxies with velocity dispersion measurements.

\begin{tabular}{|c|c|c|c|c|c|c|c|c|}
\hline $\begin{array}{l}\text { Name } \\
\text { (1) }\end{array}$ & $\begin{array}{l}m_{V} \\
(2)\end{array}$ & $\begin{array}{c}z \\
(3)\end{array}$ & $\begin{array}{l}\sigma_{\mathrm{c}} \\
(4)\end{array}$ & $\begin{array}{l}\Delta \sigma \\
(5)\end{array}$ & $\begin{array}{c}\left\langle\mu_{\mathrm{b}}\right\rangle \\
(6)\end{array}$ & $\begin{array}{c}\Delta\left\langle\mu_{\mathrm{b}}\right\rangle \\
(7)\end{array}$ & $\begin{array}{l}R_{\mathrm{b}} \\
(8)\end{array}$ & $\begin{array}{l}\Delta \Delta r_{\mathrm{b}} \\
(9)\end{array}$ \\
\hline $0648+27^{+}$ & 13.82 & 0.0409 & 137.8 & 38.0 & 14.94 & 0.05 & 0.26 & 0.02 \\
\hline $0755+37$ & 13.80 & 0.0413 & 291.0 & 17.3 & 17.05 & 0.15 & 1.09 & 0.09 \\
\hline $0908+37$ & 15.67 & 0.1040 & 387.1 & 18.0 & 17.36 & 0.10 & 0.50 & 0.05 \\
\hline $0915+32 B$ & 15.20 & 0.0620 & 252.1 & 24.3 & 17.00 & 0.05 & 0.52 & 0.03 \\
\hline $0924+30$ & 13.32 & 0.0266 & 243.7 & 14.5 & 17.33 & 0.15 & 0.96 & 0.14 \\
\hline $1003+26$ & 15.47 & 0.1165 & 438.4 & 21.6 & 17.94 & 0.15 & 0.41 & 0.05 \\
\hline $1113+24$ & 15.10 & 0.1021 & 377.8 & 10.2 & 17.44 & 0.05 & 0.47 & 0.03 \\
\hline $1204+34$ & 15.35 & 0.0788 & 201.0 & 29.6 & 17.47 & 0.25 & 0.46 & 0.09 \\
\hline $1322+36 \mathrm{~B}$ & 12.84 & 0.0175 & 259.1 & 12.1 & 15.45 & 0.05 & 0.62 & 0.05 \\
\hline $1339+26 B$ & 15.10 & 0.0757 & 379.1 & 18.2 & 16.41 & 0.15 & 0.32 & 0.04 \\
\hline $1347+28$ & 15. & 0.0724 & 228.0 & 19.4 & 17.18 & 0.05 & 0.34 & 0.03 \\
\hline $1357+28$ & 14.81 & 0.0629 & 308.3 & 11.2 & 16.80 & 0.05 & 0.40 & 0.03 \\
\hline $1422+26 B$ & 14.32 & 0.0370 & 264.9 & 11.6 & 15.44 & 0.05 & 0.13 & 0.01 \\
\hline $1430+25$ & 16.65 & 0.0813 & 203.6 & 14.3 & 16.72 & 0.25 & 0.13 & 0.03 \\
\hline $1447+27$ & 13.88 & 0.0306 & 318.4 & 11.6 & 15.77 & 0.15 & 0.47 & 0.04 \\
\hline $1450+28$ & 16.31 & 0.1203 & 405.6 & 23.6 & 16.18 & 0.05 & 0.16 & 0.15 \\
\hline $1450+28^{*+}$ & 16.31 & 0.1265 & 333.3 & 22.1 & 16.56 & 0.05 & 0.20 & 0.01 \\
\hline $1502+26$ & 15.42 & 0.0540 & 402.1 & 19.9 & 16.60 & 0.15 & 0.66 & 0.07 \\
\hline $1512+30$ & 15.37 & 0.0931 & 330.2 & 25.6 & 16.55 & 0.05 & 0.34 & 0.03 \\
\hline $1521+28$ & 15.09 & 0.0825 & 323.0 & 11.8 & 18.02 & 0.15 & 0.85 & 0.06 \\
\hline $1527+30$ & 15.64 & 0.1143 & 448.0 & 23.0 & 16.76 & 0.10 & 0.34 & 0.03 \\
\hline $1553+24$ & 14.41 & 0.0426 & 269.9 & 10.9 & 16.41 & 0.05 & 0.45 & 0.03 \\
\hline $7+26$ & 14.97 & 0.0442 & 310.7 & 16.1 & 15.45 & 0.05 & 0.21 & 0.01 \\
\hline & .90 & & 46.6 & 13.5 & 16.74 & 0.15 & 0.30 & 0.03 \\
\hline & .47 & & 315.8 & 10.2 & 15.02 & 0.15 & 0.19 & 0.02 \\
\hline & 17.04 & & & 26.8 & 15.96 & 0.15 & 0.22 & 0.03 \\
\hline & & & & 11.4 & 16.24 & 0.05 & .21 & 0.01 \\
\hline & 12.5 & 0.02 & 297.8 & 12.5 & 17.09 & 0.05 & 1.28 & 0.11 \\
\hline $0034+25$ & 13.35 & 0.03 & 295 & 10.0 & 16.27 & 0.05 & 0.74 & 0.04 \\
\hline & 0 & & 231 & 13.0 & 16.34 & 0.05 & 0.40 & 0.02 \\
\hline $0120+33$ & 11.43 & & 315 & 10.0 & 15.13 & 0.05 & 0.75 & 0.07 \\
\hline $1217+29$ & 10.41 & 0.0022 & 238.0 & 10.0 & 13.87 & 0.05 & 0.80 & 0.06 \\
\hline $1256+28$ & 13.88 & 0.0229 & 203.0 & 10.0 & 16.48 & 0.15 & 0.70 & 0.07 \\
\hline $1257+28$ & 11.94 & 0.0241 & 278.0 & 10.0 & 17.97 & 0.10 & 2.55 & 0.01 \\
\hline $1525+29$ & 14.95 & 0.0652 & 250.0 & 30.0 & 16.56 & 0.15 & 0.53 & 0.05 \\
\hline $1610+29$ & 13.29 & 0.0318 & 331.0 & 26.0 & 16.49 & 0.05 & 0.95 & 0.06 \\
\hline $3 \mathrm{C} 88$ & 14.24 & 0.0302 & 190.0 & 22.0 & 17.47 & 0.05 & 0.98 & 0.09 \\
\hline 3C192 & 15.72 & 0.0597 & 199.0 & 23.0 & 14.34 & 0.15 & 0.09 & 0.01 \\
\hline 3C 388 & 14.77 & 0.0917 & 365.0 & 23.0 & 18.03 & 0.15 & 0.96 & 0.09 \\
\hline
\end{tabular}

Columns: (1) Identification of the source, (2) apparent magnitude $m_{V}$ from Colla et al. (1975) (photographic magnitudes converted to visual magnitudes, Fanti et al. (1978), Gonzalez-Serrano et al. (2000), and Gonzalez-Serrano \& Carballo (2000), (3) redshift, (4, 5) measured velocity dispersion and error in $\mathrm{km} \mathrm{s}^{-1},(8,9),\left\langle\mu_{\mathrm{b}}\right\rangle$ and error in mag/arcsec ${ }^{2},(8,9)$ break radius $R_{\mathrm{b}}$ and error in arcsecs, from 2005; *companion of the radio galaxy, + not used in the CFP see Appendix A.1.

obtained with different template stars turned out to be less than $20 \mathrm{~km} \mathrm{~s}^{-1}$ for $\sigma$ and $\sim 10 \mathrm{~km} \mathrm{~s}^{-1}$ for the systemic radial velocity $V_{\mathrm{r}}$ both for the sample of radio galaxies than for the template galaxy. For NGC 3377 we found $\sigma=160 \pm 25 \mathrm{~km} \mathrm{~s}^{-1}$ in agreement with the value of $139 \mathrm{~km} \mathrm{~s}^{-1}$ reported in LEDA. The average values of individual determinations were adopted as final values of $\sigma$ and $V_{\mathrm{r}}$. In order to improve the $\mathrm{S} / \mathrm{N}$ ratio of the spectra, we co-added the central spectra within an aperture of $6^{\prime \prime}$. Since early-type galaxies exhibit some gradients in the radial velocity and velocity dispersion, the derived central parameter $\sigma$ depends on the distance of the galaxies and on the size of the aperture used for the observation. In order to compare our velocity dispersions with the data available in the literature, we applied aperture corrections according to the procedure given by Jørgensen et al. (1996). The individual measurements of $\sigma$ are corrected to a circular aperture with a metric diameter of $1.19 h^{-1} \mathrm{kpc}$, equivalent to $3.4^{\prime \prime}$ at the distance of the Coma cluster of galaxies.
All these data are listed in Table 1, in which we also give photometric data derived from HST images in the $F 814 W$ filter (all taken with an exposure time of $300 \mathrm{~s}$ ): as described in de Ruiter et al. (2002) the one-dimensional brightness profiles determined with the IRAF task ELLIPSE were fitted with a Nuker law, thus obtaining for each galaxy, in the $F 814 W$ (I) filter, the core radius $R_{\mathrm{b}}$ in arcsecs and the average surface brightness $\left\langle\mu_{\mathrm{b}}\right\rangle$ (in $\mathrm{mag} / \operatorname{arcsec}^{2}$ ) within the core radius.

For some galaxies the $\mathrm{S} / \mathrm{N}$ is high enough to study the inner part of the rotation curve (see Fig. A.1). In Appendix A, comments on some individual objects are reported.

\section{The CFP of radio and normal ellipticals}

To complete our data-set we decided to collect all the available data in the literature for the core properties of E galaxies. We added to the RG data the core photometric data and the velocity dispersion for two sets of data of non-radio galaxies. The 


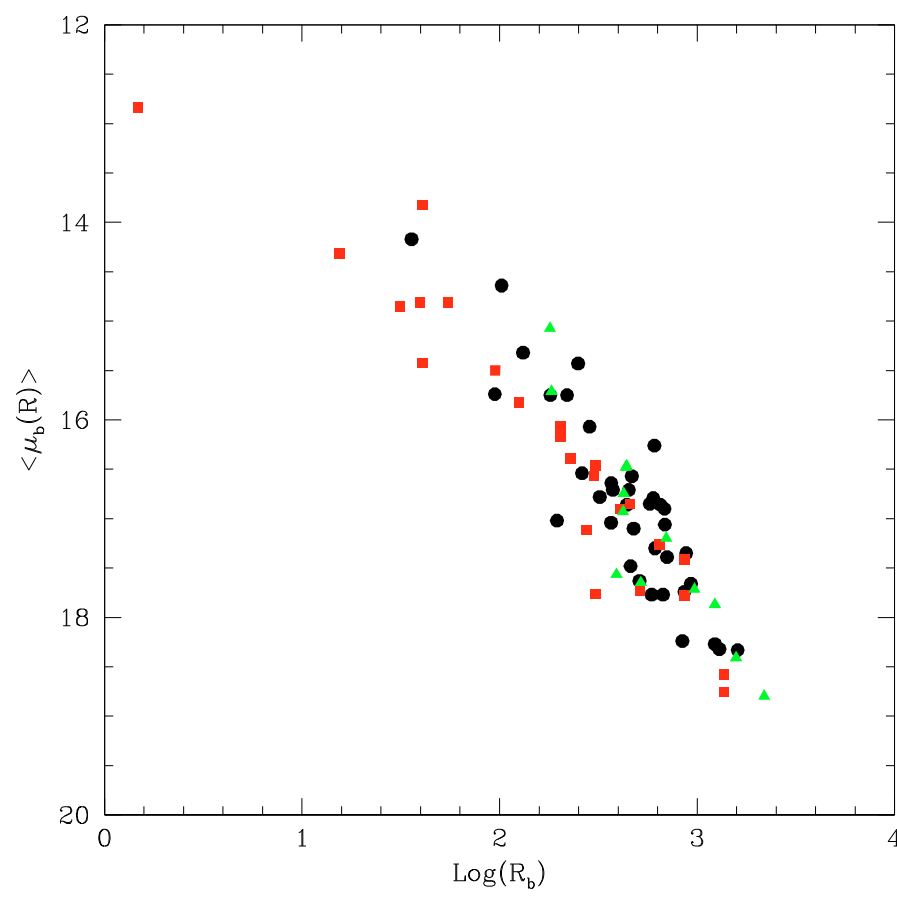

Fig. 1. The $\left\langle\mu_{\mathrm{b}}\right\rangle-R_{\mathrm{b}}$ relation of B2 radio galaxies ([black] circles) and nearby (non-radio) early type galaxies ([red] squares Faber et al. 1997; [green] triangles Bernardi et al. 2008).

first set is from the classical work of Faber et al. (1997) which include data for 24 normal core galaxies. To compare these measurements with our data for RG (derived in the $R$ band) we applied a mean color correction $V-R=0.60$. The second set is taken from the recently published HST photometric data (Hyde et al. 2008) and the corresponding central velocity dispersion $\sigma$ (Bernardi et al. 2008) for the core of 13 nearby non-radio massive galaxies.

The data of Hyde et al. (2008) were obtained using the high resolution channel (HRC) of the ACS in the $i$ band, thus we transformed their CTPS to $\mathrm{mag} / \mathrm{sq} / \mathrm{arcs}$ using the zero point for the i filter $Z P=25.654$ ( $A B$ mag). Then we applied a correction $\Delta m=-0.53$ to transform the data to the Vega magnitude system. In Fig. 1 we plot the $\left\langle\mu_{\mathrm{b}}\right\rangle-R_{\mathrm{b}}$ projection of the CFP for our sample of radio galaxies, and for a combined sample of non-radio ellipticals (Faber et al. 1997; Bernardi et al. 2008), we confirm the same correlation already found by Faber et al. (1997).

In order to derive the parameters $(\alpha, \beta$ and $\gamma)$ describing the CFP in the following relation:

$\log R_{\mathrm{b}}=\alpha \log \sigma+\beta\left\langle\mu_{\mathrm{b}}\right\rangle+\gamma$,

we minimized the root square of the residual perpendicular to the plane. In Table 2 we report the coefficients obtained using this fitting procedure for the CFP of both radio galaxies and normal ones; for comparison we report also the coefficients for the FP for radio and normal galaxies (Bettoni et al. 2001).

In Fig. 2 we show the comparison of the CFP plotted with the coefficients of our best fit and with the coefficients of the best fit of FP (Bettoni et al. 2001). The two planes are very similar and almost parallel (see also Table 2). We note a slight tendency of CFP to show a curvature at low luminosity; this is similar to what was pointed out by Desroches et al. (2007, for the FP).

In Fig. 3 we compare the whole CFP with the FP derived from the global properties of a different sample of radio and non-radio ellipticals (see Bettoni et al. 2001, for details). Again
Table 2. Coefficients of the best fit for the core fundamental plane and the global fundamental plane of low redshift radio galaxies (see Eq. (1)) and of normal galaxies.

\begin{tabular}{cccc|ccc}
\hline \hline & \multicolumn{3}{c}{ Radio Galaxies } & \multicolumn{3}{c}{ Normal Galaxies } \\
\hline & $\alpha$ & $\beta$ & $\gamma$ & $\alpha$ & $\beta$ & $\gamma$ \\
\hline CFP & 1.35 & 0.36 & -6.80 & 1.32 & 0.36 & -6.75 \\
FP & 1.24 & 0.33 & -5.96 & 1.27 & 0.32 & -5.58 \\
\hline
\end{tabular}

the CFP and FP are closely parallel, as suggested by Faber et al. (1997): whatever differences exist among core galaxies these are not so large as to erase the appearance of a two-parameter family of self-gravitating cores that is similar to the 2-dimensional family of isothermal spheres.

\section{Conclusions}

We have presented the photometric, structural and kinematic properties of the centers of a sample of 38 low redshift radio galaxies galaxies and have shown that the conventional parameters characterizing the centers (the break radius, its surface brightness and the central velocity dispersion) are well represented in a plane (the core fundamental plane) which is indistinguishable from that of elliptical galaxies that do not exhibit radio emission.

A similar result was found from the comparison of the global properties of a sample of 72 radio galaxies and local ellipticals using the standard fundamental plane description (Bettoni et al. 2001). The comparison of the properties of the FP that refer to the whole galaxy with those concering the centers (the CFP) shows that the slopes of the two planes are very similar and suggest that the same mechanism is responsible for the link between the involved quantities.

The remarkable similarity of the properties of radio and non radio elliptical galaxies in the description of both the fundamental plane and the core fundamental plane indicates that the active phase of the galaxy connected with the strong emission at radio frequencies likely has an inconsequential effect on the structure of the whole galaxy. Moreover these results suggest that the two type of galaxies (radio and non-radio) have had a similar history of formation and evolution.

\section{Appendix A: Notes on individual galaxies}

B2 0648+27 - This galaxy shows a typical E+A spectrum indicating the presence of a young stellar population (Emonts et al. 2006; Emonts et al. 2008). In this case the measured velocity dispersion is only indicative having been measured using an imperfect stellar template. This galaxy was not used to construct the CFP of RG. The spectrum show the [OII] $\lambda 3727 \AA$ emission line. For this object we were able to measure the rotation curve (both for gas and stars) and the velocity dispersion profile in the inner $\sim 10^{\prime \prime}$, these are shown in Fig. A.1. We found a $V_{\max }=200 \mathrm{~km} \mathrm{~s}^{-1}$ for both components.

B2 0908+37 - As noted by Capetti et al. (2000) a fainter companion galaxy is located $\sim 3.7^{\prime \prime}$ to the SW. Our spectrum crosses on the faint companion for which we were able to measure a redshift $z_{\text {comp }}=0.1027$, very close to the redshift of the radio galaxy.

B2 0924+30 - For this galaxy we measure a rotation $V_{\max }=$ $100 \mathrm{~km} \mathrm{~s}^{-1}$ in the inner $\sim 5^{\prime \prime}$ (see Fig. A.1). 

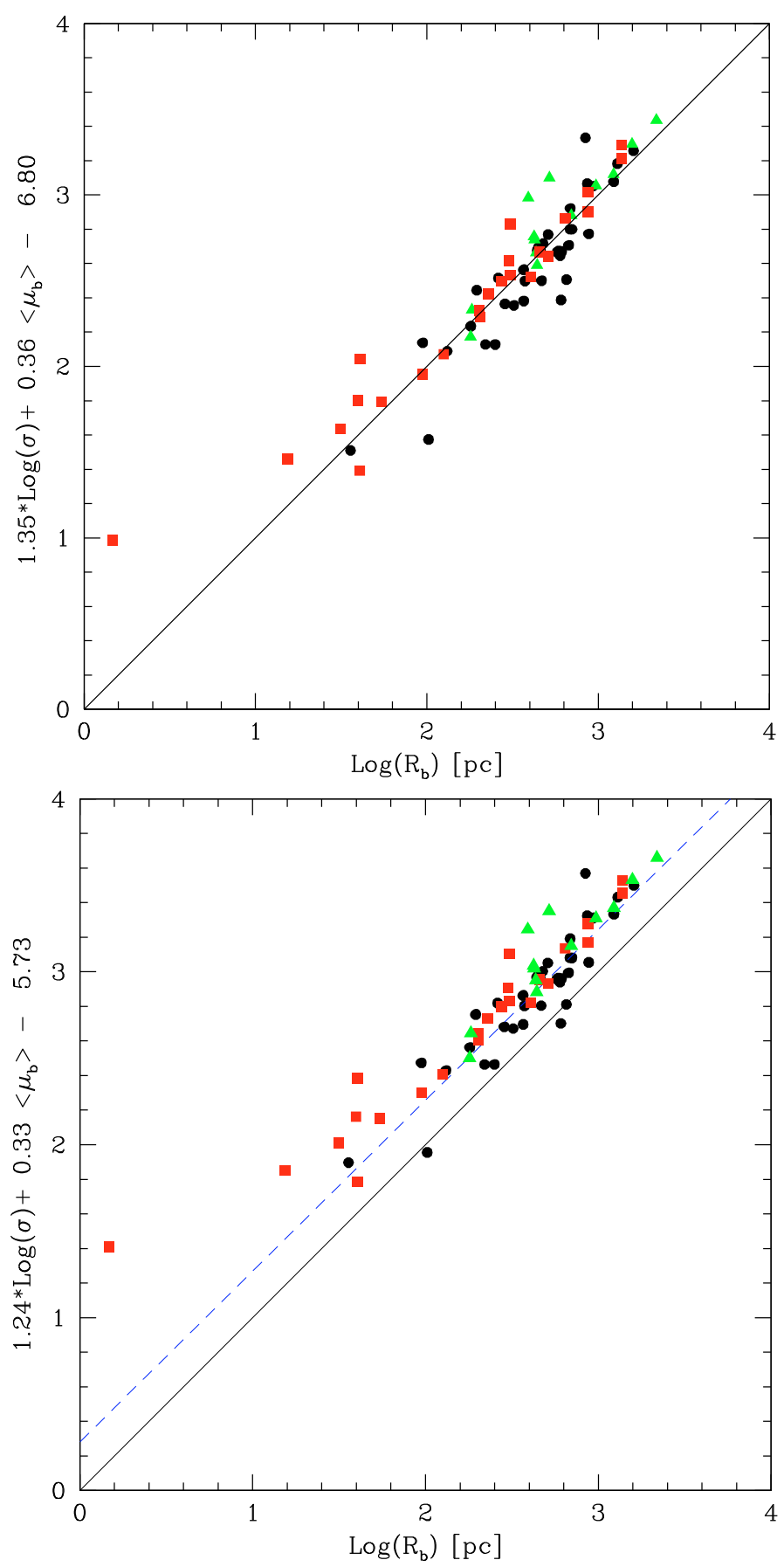

Fig. 2. Upper panel: the best fit of the CFP for the B2 radio galaxies (black circles) compared with a sample of nearby (non radio) early type galaxies ([red] squares Faber et al. 1997; [green] triangles Bernardi et al. 2008), using the CFP fit in the $y$-axis. The solid line represents CFP relation. Lower panel: The same data as in the upper panel, but using the coefficient of the global FP fit of Bettoni et al. (2001) with a shift in the $y$-axis to take into account the different units on the $x$-axis (from kpc to pc); the solid line is the corresponding FP relation. The blue dashed line reports the fit to the core data with a shift of 0.23 dex.

B2 $1113+24-$ For this galaxy we measure a rotation $V_{\max }=$ $180 \mathrm{~km} \mathrm{~s}^{-1}$ in the inner $\sim 5^{\prime \prime}$ (see Fig. A.1).

B2 1204+34 - This galaxy show strong emission lines in the spectrum i.e [O III] $\lambda 5007 \AA$ and $\mathrm{H}_{\beta}$. We measured the gas rotation curve, and after subtraction of the emission component, also the stellar rotation curve (see Fig. A.1). The gas is more extended in the NE direction and show a $V_{\max } \sim 200 \mathrm{~km} \mathrm{~s}^{-1}$.

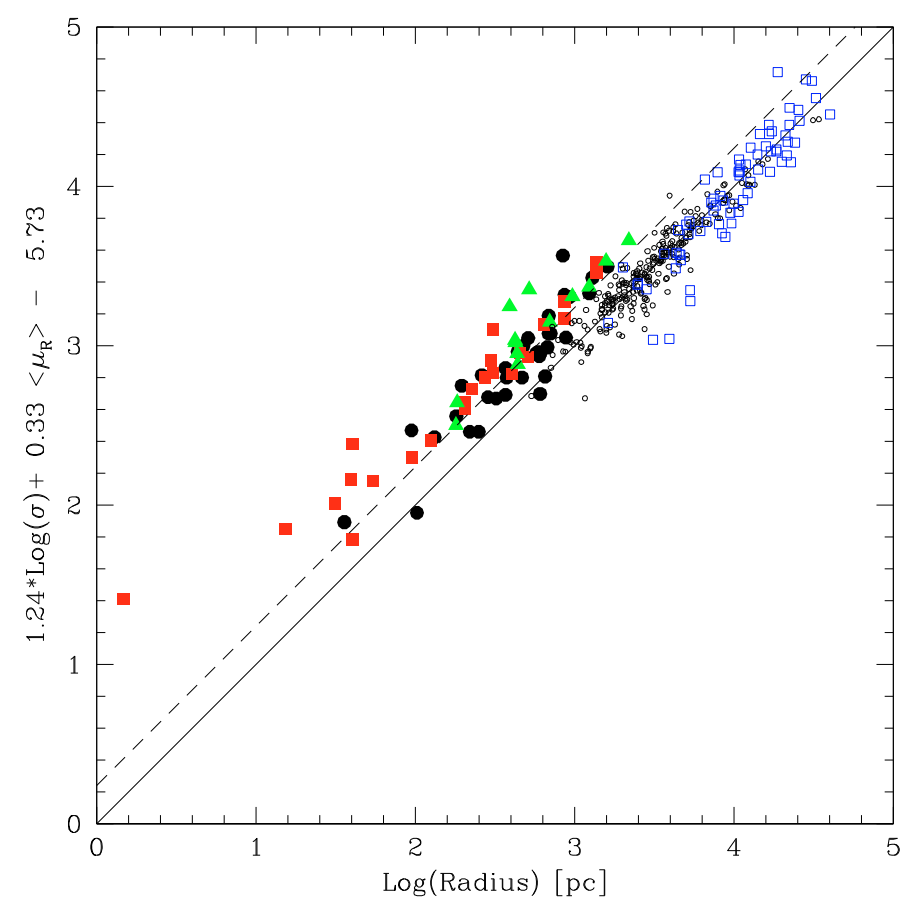

Fig. 3. The CFP of B2 radio galaxies (black circles) and nearby (non radio) early-type galaxies ([red] squares Faber et al. 1997; [green] triangles Bernardi et al. 2008) compared to the Fundamental Plane of normal small black dots (Jørgensen et al. 1996) and radio galaxies lightblue squares (Bettoni et al. 2001). The solid line represents the FP for a sample of RG (Bettoni et al. 2001) while the dashed line yields the fit to the core data. Note that we plot $R_{\mathrm{b}}$ and $\left\langle\mu_{\mathrm{b}}\right\rangle$ for the CFP data (black circles, red squares and green triangles) and $R_{\mathrm{e}}$ and $\left\langle\mu_{\mathrm{e}}\right\rangle$ for the FP data (small black open circles and small blue open squares).

B2 $1322+36$ - NGC 5141 - This S0 galaxy has a nuclear dust lane and the gas exhibits a regular rotation profile as measured by Noel-Storr et al. (2003). Here we measure, for the first time, a regular rotation curve for the stellar component with a $V_{\max }=200 \mathrm{~km} \mathrm{~s}^{-1}$ at $r=\sim 10^{\prime \prime}$ (see Fig. A.1). Our spectra are in the region $\lambda 3800-4800 \AA$ where only a very faint $\lambda 3727$ [OII] line is visible; the gas and stellar components are co-rotating.

B2 $1422+26 \mathrm{~B}-$ This galaxy show the presence in the spectrum of the [O II] $\lambda \lambda 3727 \AA$ line.

B2 $1450+28$ - This galaxy is a well known dumbbell system in the center of the cluster Abell 1984. The radio galaxy is associated with the southern galaxy of the pair. To obtain our spectrum, the slit was oriented at $\mathrm{PA}=0^{\circ}$ and crosses both galaxies. In Table 1 we report the velocity dispersion for both objects. In Table 1 the non-radio galaxy is labelled with an asterisk and was excluded from the fit.

B2 $1502+26$ - 3C 310 - This galaxy shows a boxy elliptical galaxy approximately $15^{\prime \prime} \mathrm{SW}$, the slit for our spectrum was oriented at $\mathrm{PA}=90^{\circ}$ and crosses both galaxies, for the fainter companion we measure the same redshift $z=0.0540$ and a velocity dispersion $\sigma=390.0 \pm 22.7 \mathrm{~km} \mathrm{~s}^{-1}$.

B2 1527+30 - in Abell 2083; this galaxy show a strong velocity gradient $V_{\max }=180 \mathrm{~km} \mathrm{~s}^{-1}$ in the inner $10^{\prime \prime}$ (see Fig. A.1).

Acknowledgements. We thanks the anonymous referee whose comments helped us to improve our manuscript. This research made use of Vizier service (Ochsenbein et al. 2000) and of the NASA/IPAC Extragalactic Database (NED) which is operated by the Jet Propulsion Laboratory, California Institute of Technology, under contract with the National Aeronautics and Space 

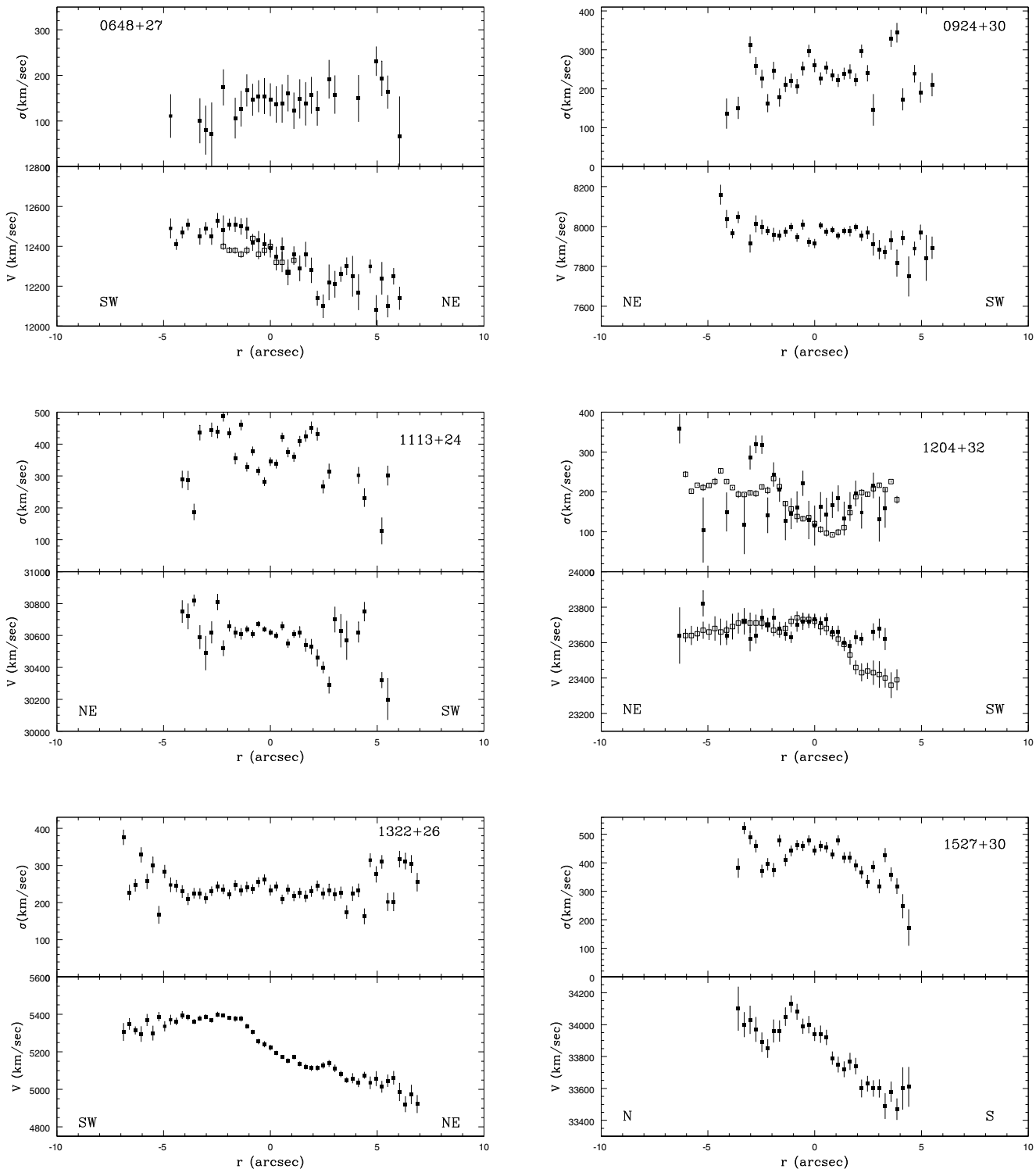

Fig. A.1. Rotation curves and velocity dispersion profiles for selected low redshift radio galaxies (full squares stars, open squares gas).

Administration. We have made use of the LEDA (http: //leda. univ-lyon1 fr) and Hypercat database.

\section{References}

Balmaverde, B., \& Capetti, A. 2006, A\&A, 447, 97

Bernardi, M., Hyde, J. B., Fritz, A., et al. 2008, MNRAS, 391, 1191

Bettoni, D., Falomo, R., Fasano, G., et al. 2001, A\&A, 380, 471

Bertola F., Bettoni, D., Rusconi, L., \& Sedmak, G. 1994, AJ, 89, 356

Capetti, A., \& Balmaverde, B. 2006, A\&A, 453, 27

Capetti, A., \& Celotti, A. 1999, MNRAS, 304, 434

Capetti, A., de Ruiter, H. R., \& Fanti, R. 2000, A\&A, 362, 871

Colla, G., Fanti, C., Fanti, R., et al. 1975, A\&AS, 20, 1

Desroches, L.-B., Quataert, E., Ma, C.-P., \& West, A. A. 2007, MNRAS, 377, 402

De Koff, S., Baum, S. A., Sparks, W. B., et al. 1996, ApJS, 107, 621

De Juan, L., Colina, L., \& Golombek, D. 1996, A\&A, 305, 776

de Ruiter, H. R., Parma, P., Fanti, C., \& Fanti, R. 1990, A\&A, 227, 351

de Ruiter, H., Parma, P., Capetti, A., Fanti, R., \& Morganti, R. 2002, A\&A, 396, 857

de Ruiter, H. R., Parma, P., \& Capetti, A. 2005, A\&A, 439, 487

Emonts, B. H. C., Morganti, R., Tadhunter, C. N., et al. 2006, A\&A, 454, 125

Emonts, B. H. C., Morganti, R., van Gorkom, J. H., et al. 2008, A\&A, 488, 519

Faber, S. M., Tremaine, S., Ajhar, E. A., et al. 1997, ApJ, 114, 5
Fanti, R., Gioia, I., Lari, C., \& Ulrich, M. H. 1978, A\&AS, 34, 341 Fanti, C., Fanti, R., De Ruiter, H. R., \& Parma, P. 1987, A\&AS, 69, 57 Ferrarese, L., \& Merritt, D. 2000, ApJ, 539, L9

González-Serrano, J. I., \& Carballo, R. 2000, A\&AS, 142, 353

González-Serrano, J. I., Carballo, R., \& Perez-Fournon 1993, AJ, 105, 1710

Jaffe, W., Ford, H. C., Ferrarese, L., Van den Bosch, F., \& O'Connell, R.W. 1993, Nature, 364, 213

Jørgensen, I., Franx, M., \& Kjaergaard, P. 1996, MNRAS, 280, 167

Hyde, J. B., Bernardi, M., Sheth, R. K., \& Nichol, R. C. 2008, MNRAS, 391, 1559

Kormendy, J., Fisher, D. B., Cornell, M. E., \& Bender, R. 2009, ApJS, 182, 21

Lauer, T. R., Faber, S. M., Richstone, D., et al. 2007, ApJ, 662, 808

Massaglia, S., Trussoni, E., Caucino, S., et al. 1996, A\&A, 309, 75

Morganti, R., Fanti, R., Gioia, I. M., et al. 1988, A\&A, 189, 11

Morganti, R., Parma, P., Capetti, A., et al. 1997, A\&AS, 126, 335

Noel-Storr, J., Baum, S. A., Verdoes Kleijn, G., et al. 2003, ApJS, 148, 419

Ochsenbein, F., Bauer, P., \& Marcout, J. 2000, A\&ASS, 143, 23

Parma, P., Fanti, C., Fanti, R., Morganti, R., \& De Ruiter, H. R. 1987, A\&A, 181, 244.

Paturel, G., Andernach, H., Bottinelli, L., et al. 1997, A\&AS, 124, 109

Sargent, W. L. W., Schechter, P. L., Boksenberg, A., \& Shortridge, K. 1977, ApJ, 212,326

Trussoni, E., Massaglia, S., Ferrari, R., et al. 1997, A\&A, 327, 27

Verdoes Kleijn, G. A., Baum, S. A., de Zeeuw, P. T., \& O’Dea, C. P. 1999, AJ, 118,2592 